\title{
Separating Lambertian and Specular Reflectance Components using Iterated Conditional Modes
}

\author{
Hossein Ragheb Edwin. R. Hancock \\ Department of Computer Science, \\ University of York, York YO1 5DD, UK. \\ hossein,erh@minster.cs.york.ac.uk
}

\begin{abstract}
In this paper we address the problem of separating Lambertian and specular reflection components in order to improve the quality of surface normal information recoverable using shape-from-shading. The framework for our study is provided by the iterated conditional modes algorithm. We develop a maximum a posteriori probability (MAP) estimation method for estimating the mixing proportions for Lambertian and specular reflectance, and also, for recovering local surface normals. The MAP estimation scheme has two model ingredients. Firstly, there are separate conditional measurement densities which describe the distributions of surface normal directions for the Lambertian and specular reflectance components. The second ingredient is a smoothness prior which models the distribution of surface normal directions over local image regions. We experiment with the method on real-world data. This reveals not only that the method accurately estimates the proportion of specular reflection, but that it also results in good surface normal reconstruction in the proximity of specular highlights.
\end{abstract}

\section{Introduction}

Shape-from-shading is concerned with recovering surface orientation from local variations in measured brightness. There is strong psychophysical evidence for its role in surface perception and recognition $[1,13,11]$.

The observation underpinning this paper is that although considerable effort has gone into the recovery of accurate surface geometry, existing shape-from-shading methods are confined to situations in which the reflectance is predominantly Lambertian. When local specularities are present, then the estimated geometry may be subject to error. The main problem that can occur is that specular highlights may be misidentified as curved surface features such as ridges and domes. Nonetheless, if specular highlights can be accurately located, then they can provide important cues that can be used to constrain the recovery of surface shape. For instance, Healey and Binford [9] have shown how a simplified version of the Torrance and Sparrow model [19] can be used to analyse surface curvature. In a comprehensive treatment of specular shape-from-shading Brelstaff and Blake [3, 5] have analysed the geometric constraints provided by specularities, and have shown how to 
detect specularities using Lambertian irradiance constraints. Drawing on psychophysics, Blake and Bulthoff [4] have developed a computational model of the shape information available to a moving observer from the apparent movement of specularities. Several authors have looked critically at the physics underlying specular reflectance. For instance, Nayar, Ikeuchi and Kanade [17] have shown that the Torrance and Sparrow model [19] is applicable to the modelling of the specular lobe rather than the specular spike. In a series of recent papers, Lin and Lee have shown how specular reflections due to multiple lightsources can be located in multi-band imagery $[14,15,16]$. Finally, Nayar, Fang and Boult [18] have used polarisation filters to detect specular reflection. The main conclusion to be drawn from this brief review of the literature is that although specularities provide important geometric constraints for shape-from-shading, their reliable modelling and detection in single-band (i.e. grey-scale) images has proved an elusive goal.

Our aim in this paper is to develop a Bayesian framework for recovering shape-fromshading in the presence of local specular highlights. We adopt a maximum a posteriori probability estimation framework using the iterative conditional modes algorithm of Besag [2]. The aim is to estimate the proportions of Lambertian and specular reflectance, and hence recover more accurate surface normal directions. To meet this goal we must provide probability distributions for the surface normals associated with the observed image intensity. We develop a geometric framework and concentrate on modelling the angular distributions for the surface normals resulting from the two reflectance components. In the case of Lambertian reflectance, the surface normal is constrained to fall on a cone whose axis is aligned in the light source direction and whose apex angle is determined by the observed image brightness. We assume that angular deviations from this reflectance cone follow a Gaussian distribution. For specular reflectance, the surface normal direction is the bisector of the viewer and light source directions. Angular deviations for the surface normals associated with the specular spike are assumed to follow a Beckmann distribution. At each image location, the Bayes framework allows us to estimate the proportions of Lambertian and specular reflectance. With this information to hand we can compute the weighted mean of the ideal Lambertian and specular reflectance directions, and hence obtain the posterior mean surface normal direction.

From the posterior mean surface normal direction, we can reconstruct the specular reflectance using the Torrance and Sparrow model. This allows us to subtract specular hightlights from the original image. By re-applying the shape-from-shading technique to the residual matte reflectance component, we are able to extract better surface normal information which is free of the spurious curvature artifacts associated with specular highlights. Experiments on real world images reveal promising results.

\section{Reflectance Model}

In this section we outline the geometry of the reflectance processes which underpin our shape-from-shading model. We adopt a two-component model in which the predominantly Lambertian surface reflectance exhibits local specular highlights.

\subsection{Lambertian Reflectance}

In the case of Lambertian reflectance from a matte surface of constant albedo illuminated with a single collimated light-source, the observed intensity is independent of the viewing 
direction. The observed intensity depends only on the quantity of absorbed light, and this in turn is proportional to the cosine of the incidence angle between the light source direction and the local normal vector to the surface. Suppose that $\vec{L}$ is the unit-vector in the direction of the light source and that $\vec{N}_{L}$ is the unit-vector in the surface normal direction. According to Lambert's law, the observed image intensity at the pixel with co-ordinates $(i, j)$ is $E(i, j)=\vec{N}_{L}(i, j) \cdot \vec{L}$

Lambert's equation provides insufficient information to uniquely determine the surface normal direction. However, the equation does have a simple geometric interpretation which can be used to constrain the direction of the surface normal. The equation specifies that the surface normal must fall on the surface of a right-cone whose axis is aligned in the light-source direction $\vec{L}$ and whose apex angle is $\cos ^{-1} E(i, j)$. This property has recently been exploited by Worthington and Hancock [21] to develop a two-step iterative process for shape-from-shading. The process commences from a configuration in which the surface normals are placed on the position on the irradiance cone where their projections onto the image plane are aligned in the direction of the local (Canny) image gradient.

In the first step, the surface normal directions are subjected to smoothing in such a way as to satisfy curvature consistency constraints. The resulting smoothed surface normal $\hat{\vec{N}}_{L}^{(n)}$ will not fall on the irradiance cone and will hence not satisfy Lambert's law. To overcome this problem the smoothed surface normal is rotated onto the nearest location on the irradiance cone. The resulting surface normal, which satisfies Lambert's law, is $\vec{N}_{L}^{(n+1)}=\hat{\vec{N}}_{L}^{(n)} \cos (\theta)+\left(\vec{R} \times \hat{\vec{N}}_{L}^{(n)}\right) \sin (\theta)$ where the rotation axis is given by $\vec{R}=\frac{\hat{\vec{N}}_{L}^{(n)} \times \vec{L}}{\left\|\hat{\vec{N}}_{L}^{(n)} \times \vec{L}\right\|}$ and the rotation angle is $\quad \theta=\cos ^{-1}\left(\hat{\vec{N}}_{L}^{(n)} \cdot \vec{L}\right)-\cos ^{-1}(E)$.

\subsection{Specular Reflectance}

The second component of our reflectance process is concerned with modelling local specular highlights on the observed surface. For specular reflection the surface normal, the light source direction and the viewing direction are coplanar. The incidence angle (i.e. the angle between the surface normal and the light source direction) is equal to the angle of specular reflectance (i.e. the angle between the surface normal and the viewing direction). Hence, for specular reflection, the direction of the surface normal $\vec{N}_{S}^{(n)}$ is the bisector of the light source direction $(\vec{L})$ and the viewing direction $(\vec{V})$ and the unit-vector is $\quad \vec{N}_{S}^{(n)}=\frac{(\vec{L}+\vec{V})}{\|\vec{L}+\vec{V}\|}$.

It is important to stress that the surface normal for the specular reflectance component is fully constrained if the light source direction and the viewing direction are known.

\section{Bayesian Framework}

The aim in this paper is to develop a Bayes-decision scheme for classifying pixels according to the two reflectance modes described in the previous section. In other words, we wish to compute the a posteriori probabilities of specular or Lambertian reflectance. To meet this goal we make use of the iterated conditional modes algorithm of Besag [2]. Although the method has notoriously poor global optimisation properties, we use it here 
because it is simple and relatively efficient. In this paper our primary aim is to investigate the feasibility of applying probabilistic methods to the shape-from-shading problem. The use of more sophisticated optimisation methods such as simulated annealing, Markov chain Monte-Carlo or mean field annealing will be the focus of future work.

The aim is to label pixels according to the reflectance mode from which they originated. The class assignment for the pixel $(i, j)$ at iteration $n$ is denoted by $\omega_{i, j}^{(n)}$. The assignment may be drawn from the set $\Omega=\{S, L\}$ where $S$ is the specular reflectance label and $L$ is the Lambertian reflectance label. For each image location, we maintain a specular surface normal and a Lambertian surface normal which satisfy the geometric constraints outlined in Section 2. At iteration $n$ of the algorithm the currently available estimates of the Lambertian and specular surface normals at the pixel indexed $(i, j)$ are respectively $\vec{N}_{L}^{(n)}(i, j)$ and $\vec{N}_{S}^{(n)}(i, j)$. In the case of the specular component, the normal direction is in the direction of local specular reflection, i.e. the bisector of the light source and viewing directions, and does not change with iteration number. In the case of Lambertian reflectance, the surface normal direction varies with iteration number, but is always projected to be positioned on the irradiance cone.

To develop our decision process, we require two probabilistic modelling ingredients. The first of these are separate probability density functions which can be used to represent the distributions of surface normal directions for the specular and Lambertian reflectance components. We evaluate these densities at the posterior mean surface normal $\vec{M}^{(n)}(i, j)$ computed at iteration $n$. The reason for doing this is that the current values of the specular and Lambertian normals are guaranteed to satisfy the geometric constraints outlined in Section 2. As a result, they will be associated with vanishing angular error. Accordingly, we let $p\left(\vec{M}^{(n)}(i, j) \mid \omega_{i, j}^{(n)}=L\right)$ be the probability distribution for the posterior mean surface normal under the Lambertian reflectance model. Similarly, we let $p\left(\vec{M}^{(n)}(i, j) \mid \omega_{i, j}^{(n)}=S\right)$ denote the distribution function for the posterior mean surface normal for the specular reflectance component.

The second probabilistic ingredient is a smoothness prior for the selected surface normal direction. This component of the model incorporates contextual information. We let $P\left(\vec{N}_{L}^{(n)}(i, j) \mid \vec{M}^{(n)}(k, l), \forall(k, l) \in G_{i, j}\right)$ be the conditional probability of the Lambertian surface normal at the location $(i, j)$ given the field of surrounding posterior-mean surface normals $\vec{M}^{(n)}(k, l), \forall(k, l) \in G_{i, j}$ in the neighbourhood $G_{i, j}$ of the pixel $(i, j)$. With these ingredients, then according to the iterated conditional modes, the probability that the pixel $(i, j)$ belongs to the Lambertian class at iteration $n$ is

$$
P\left(\omega_{i, j}^{(n)}=L\right)=\frac{p\left(\vec{M}^{(n)}(i, j) \mid \omega_{i, j}^{(n)}=L\right) P\left(\vec{N}_{L}^{(n)}(i, j) \mid \vec{M}^{(n)}(k, l), \forall(k, l) \in G_{i, j}\right)}{\sum_{\Lambda \in \Omega} p\left(\vec{M}^{(n)}(i, j) \mid \omega_{i, j}^{(n)}=\Lambda\right) P\left(\vec{N}_{\Lambda}^{(n)}(i, j) \mid \vec{M}^{(n)}(k, l), \forall(k, l) \in G_{i, j}\right)}
$$

The probability that the surface normal belongs to the specular class is the complement, i.e. $P\left(\omega_{i, j}^{(n)}=S\right)=1-P\left(\omega_{i, j}^{(n)}=L\right)$. These probabilities can be used to label pixels according to the specular and Lambertian reflectance modes. With the a posteriori class probabilities to hand, we can update the estimate of the posterior-mean surface normal in the following manner

$$
\vec{M}^{(n+1)}(i, j)=\vec{N}_{S}^{(n)}(i, j) P\left(\omega_{i, j}^{(n)}=S\right)+\vec{N}_{L}^{(n)}(i, j) P\left(\omega_{i, j}^{(n)}=L\right)
$$




\section{Model Ingredients}

To apply the decision rule developed in the previous Section, we require models for the probability density functions for the distributions of specular and Lambertian surface normal directions, and also for the conditional priors for the surface normals. In this section we detail these models.

\subsection{Surface Normal Distributions}

We now provide details of the probability densities used to model the distributions of surface normal directions. In the case of the Lambertian reflectance component, the model is based on the difference of predicted and observed image intensity. In the case of the specular component, we do not use intensity information; we simply use the difference in angle between the posterior surface normal and the specular reflectance spike.

\subsubsection{Lambertian Reflectance}

Our model of the Lambertian reflectance process assumes that the observed intensity values follow a Gaussian distribution with variance $\sigma_{L}^{2}$. At the pixel indexed $(i, j)$ the mean intensity is $\vec{M}^{(n)}(i, j) \cdot \vec{L}$. Under these assumptions we can write

$$
p\left(\vec{M}^{(n)}(i, j) \mid \omega_{i, j}^{(n)}=L\right)=\frac{1}{\sqrt{2 \pi} \sigma_{L}} \exp \left[\frac{-1}{2}\left(\frac{\left.E(i, j)-\vec{M}^{(n)}(i, j) \cdot \vec{L}\right)}{\sigma_{L}}\right)^{2}\right]
$$

It must be stressed that there are alternative models to hand. For instance, Wolff and Nayer [20] have looked critically at the validity of Lambert's law and have found departures at small grazing angles to the surface. However, these models require the specification of additional parameters and hence prove to be too cumbersome for our purposes.

\subsubsection{Specular Reflectance}

The modelling of specular reflectance and specular highlights has attracted considerable attention in the computer vision and computer graphics communities. Broadly speaking there are three approaches. The model of Torrance and Sparrow [7, 19] captures the physics of scattering by the micro-facet structure of a surface. However, it is difficult to control and requires that a number of physical parameters for the surface be provided. The Beckmann model provides a simpler picture which captures the angular shape of the specular spike. Finally, Healey and Binford [9] have a simple model which can be used to model the distribution of specular intensities for regions of high surface curvature.

Here we are only interested in the distribution of surface normal directions for local specular highlights. Hence we use the Beckmann distribution to model the angle $\alpha=$ $\cos ^{-1}\left(\vec{M}^{(n)}(i, j) \cdot \vec{N}_{S}^{(n)}(i, j)\right)$, between the posterior mean surface normal $\vec{M}^{(n)}(i, j)$ and the predicted direction of the specular spike $\vec{N}_{S}^{(n)}(i, j)$ at the pixel indexed $(i, j)$.

$$
p\left(\vec{M}^{(n)}(i, j) \mid \omega_{i, j}^{(n)}=S\right)=D(\alpha)=\frac{1}{\sigma_{S}^{2} \cos ^{4} \alpha} \exp \left[-\left(\frac{\tan (\alpha)}{\sigma_{S}}\right)^{2}\right]
$$


where $\sigma_{S}$ is a parameter which controls the angular spread of the specular spike. This distribution simply models deviations of the posterior mean surface normal away from the direction of the specular spike. It makes no attempt to model either the distribution of specular intensities, nor the distribution of surface normal directions within the specular lobe.

\subsection{Smoothness Prior}

Our model for the surface normal smoothness prior is based on the average value of the inner product of the surface normal at the location $(i, j)$ with the surrounding field of surface normals. We write

$$
P\left(\vec{N}_{\Lambda}^{(n)}(i, j) \mid \vec{M}^{(n)}(k, l), \forall(k, l) \in G_{i, j}\right)=\frac{1}{2\left|G_{i, j}\right|}\left[\left|G_{i, j}\right|+\sum_{(k, l) \in G_{i, j}} \vec{N}_{\Lambda}^{(n)}(i, j) \cdot \vec{M}^{(n)}(k, l)\right]
$$

\section{Specular Shape-from-shading Algorithm}

Having described the Bayes framework and the associated two-mode reflectance model, we are now in a position to develop a practical shape-from-shading algorithm. At each image location, we maintain a specular surface normal and a Lambertian surface normal which satisfy the geometric constraints outlined in Section 2. This process draws on the contextual information provided by the surrounding field of surface normal directions.

We commence by initialising the algorithm. The initial Lambertian surface normal $\vec{N}_{L}^{(0)}(i, j)$ is constrained to lay on the irradiance cone in the direction of the image gradient. The specular surface normal is the bisector of the light source and viewing directions. The subsequent iterative steps of the algorithms are as follows:

- Using the currently available estimates of the specular and Lambertian surface normals, we compute the posterior mean surface normal $\vec{M}^{(n)}(i, j)$.

- The field of posterior mean surface normals is subjected to local smoothing. Here we use the curvature sensitive smoothing method described by Worthington and Hancock. The smoothed surface normal is denoted by $\vec{M}_{R}^{(n)}(i, j)$.

- We update the current estimate of the Lambertian surface normal direction by projecting the smoothed posterior mean direction onto the nearest location on the irradiance cone using the geometric construction outlined in Section 2.1. This gives us the revised surface normal $\vec{N}_{L}^{(n)}(i, j)$. Note that the specular surface normal direction is fully constrained and hence does not need to be updated.

- With the smoothed posterior surface normal direction to hand we compute the conditional measurement densities $p\left(\vec{M}_{R}^{(n)}(i, j) \mid \omega_{i, j}^{(n)}=L\right)$ and $p\left(\vec{M}_{R}^{(n)}(i, h) \mid \omega_{i, j}^{(n)}=\right.$ $S)$ for the Lambertian and specular reflectance modes. Taking the smoothed posterior mean surface normal directions and the current surface normal directions for the two reflectance modes, we compute the smoothness priors $P\left(\vec{N}_{S}^{(n)}(i, j) \mid \vec{M}_{R}^{(n)}(k, l), \forall(k, l) \in G_{i, j}\right)$ and $P\left(\vec{N}_{L}^{(n)}(i, j) \mid \vec{M}_{R}^{(n)}(k, l), \forall(k, l) \in\right.$ $\left.G_{i, j}\right)$. With these two ingredients to hand, we compute the updated with-context 
a posteriori probabilities for the specular and Lambertian reflectance modes, i.e. $P\left(\omega_{i, j}^{(n)}=S\right)$ and $P\left(\omega_{i, j}^{(n)}=L\right)$.

- With the a posteriori reflectance label probabilities to hand, we update the value of the posterior mean surface normal using the formula

$$
\vec{M}^{(n+1)}(i, j)=\vec{N}_{S}^{(n)}(i, j) P\left(\omega_{i, j}^{(n)}=S\right)+\vec{N}_{L}^{(n)}(i, j) P\left(\omega_{i, j}^{(n)}=L\right)
$$

This is simply the weighted sum of the unit-vectors for the specular and Lambertian reflectance components.

The steps of the algorithm are summarised in Figure 1.

\section{Specular Subtraction}

In this Section we describe how the posterior mean surface normals delivered by our shape-from-shading algorithm can be used for the purposes of reconstructing the specular intensity component. The reason for doing this is that the specular intensity may be subtracted from the original image intensity to give a corrected Lambertian image. By re-applying the shape-from-shading algorithm to this corrected intensity image, we aim to recover improved surface normal estimates, free of the high curvature artifacts of specular highlights. The process of subtracting the specular component can be viewed as equivalent to that adjusting the angles of the Lambertian reflectance cones described in Section 2.1. In principle, this subtraction process could be iterated several times. However, in practice we find that one application is sufficient to give insignificant specular probabilities over the entire image.

We use the Torrance-Sparrow model $[19,7]$ to reconstruct the specular intensity component. According to this model, the specular contribution to the image brightness is

$$
I_{S}(i, j)=\left(K \frac{F}{\pi}\right) \frac{G}{\left(\vec{M}^{(f)}(i, j) \cdot \vec{V}\right)} \frac{D(\alpha)}{\left(\vec{M}^{(f)}(i, j) \cdot \vec{L}\right)}
$$

where $\vec{M}^{(f)}(i, j)$ is the final posterior mean surface normal direction. The model is controlled by four terms. The first of these is the Fresnel term which is close to unity, i.e. $F \cong 1.0$. Secondly, there is the geometrical attenuation factor

$G=\min \left[1,2 \frac{\vec{M}^{(f)}(i, j) \cdot \vec{N}_{S}(i, j)\left(\vec{M}^{(f)}(i, j) \cdot \vec{V}\right)}{\vec{V} \cdot \vec{N}_{S}(i \cdot j)}, 2 \frac{\vec{M}^{(f)}(i, j) \cdot \vec{N}_{S}(i, j)\left(\vec{M}^{(f)}(i, j) \cdot \vec{L}\right)}{\vec{V} \cdot \vec{N}_{S}(i, j)}\right]$

Thirdly, there is the facet slope function which we model using the Beckmann distribution $D(\alpha)$. Fourthly, and finally, there is the "equaliser" term $K=\pi \sigma^{2}\left(\vec{N}_{S}(i, j) \cdot \vec{L}\right)^{2}$. With the reconstructed specular intensity to hand, we can compute the matte reflectance component

$$
I_{L}(i, j)=E(i, j)-I_{S}(i, j)
$$

The specular subtraction process is controlled by the single roughness parameter $\sigma$ of the surface. Once the corrected Lambertian intensity $I_{L}(i, j)$ is to hand, then we can use it as input to a re-application of our shape-from-shading algorithm. However, rather than initialising the algorithm with surface normal directions defined by the image gradient, we use the posterior mean surface normal directions available from the application of the shape-from-shading algorithm to the raw image intensity. 


\section{Experiments}

We have experimented with the new shape-from-shading method on a variety of real world images of shiny objects. In Figure 2 we show the results obtained for an image of a porcelain urn. The image was captured using an Olympus 10E camera and the object was illuminated with a collimated tungsten light. Here the camera viewing direction and the light source direction are separated by 20 degrees. From left to right, the images in the first row of the Figure show the original image, the specular component and the separated Lambertian reflectance component. There is strong specular reflection near the centre of the cylindrical body of the object and around the curved handles. In the image of the Lambertian reflectance component there is only slight evidence of residual specular highlight just below the ribbed structure on the surface. In the second row of Figure 2 from left to right we show the needle map extracted from the Lambertian reflectance component, the needle map extracted from the original image using the algorithm of Worthington and Hancock [21], and the field of vector differences between the two needle maps. In the case of the Worthington and Hancock algorithm, there is significant local needle-map structure around the central specularity. In the case of our new specular subtraction method, the structure is absent. From the difference-field for the two needle maps it is clear that the main structural differences occur around the handles and body of the urn. The two images in the final row of Figure 2 show the total surface curvature extracted by the new specular subtraction method (left) and the Worthington and Hancock algorithm (right). Here the specularities manifest themselves as high curvature local surface features.

Figures 3 and 4 repeat this sequence of images for details centred around the the left handle of the urn and the bright specular highlight in the centre of the urn. These images underline the observations made above. Two more sequences are shown in Figures 5 and 6 for a porcelain model of a bear and the neck of a porcelain vase. Again the specular subtraction works well.

\section{Conclusions}

In this paper, we have shown how to separate the matte and specular reflectance from grey-scale images of shiny curved objects. To do this we have developed a maximum a posteriori probability estimation framework for specular shape-from-shading. The algorithm uses separate probability density functions to model the angular distributions for the surface normals resulting from the specular and matte reflectance components. The method allows us to subtract the specular reflectance component from shiny surfaces. This allows us to recover surface shape-information which does to contain the curvature artifacts produced by local specular highlights. The method has been demonstrated to be effective on a variety of real world images.

\section{References}

[1] Belhumeur, P.N. and Kriegman, D.J. (1996) What is the Set of Images of an Object Under All Possible Lighting Conditions? CVPR, pp. 270-277.

[2] Besag, J. (1986) On the Statistical Analysis of Dirty Pictures, J. R. Statis. Soc. Lond. B, Vol. 48, pp. 259-302. 
[3] Blake, A. and Brelstaff, G. (1988) Geometry from Specularities, ICCV, pp. 394-403.

[4] Blake, A. and Bulthoff, H. (1991) Shape from Specularities: Computation and Psychophysics, Phil Trans R. Soc. Lond. B, Vol. 331, pp. 237-252.

[5] Bichsel, M. and Pentland, A.P. (1992) A Simple Algorithm for Shape from Shading, CVPR, pp. 459-465.

[6] Brelstaff, G. and Blake, A. (1988) Detecting Specular Reflection Using Lambertian Constraints, ICCV, pp. 297-302.

[7] Brooks, M.J. and Horn, B.K.P. (1986) Shape and Source from Shading, IJCAI, pp. 932-936. bibitemBruckstein88 Bruckstein, A.M. (1988) On Shape from Shading, CVGIP, Vol. 44, pp. 139-154.

[8] Cook, R.L. and Torrance, K.E. (1981) A Reflectance Model for Computer Graphics, Computer Graphics, Vol. 15, No. 3, pp. 307-316.

[9] Ferrie, F.P. and Lagarde, J. (1990) Curvature Consistency Improves Local Shading Analysis, $I C P R$, Vol. I, pp. 70-76.

[10] Healey, G.H. and Binford, T.O. (1987) Local Shape from Specularity, ICCV, pp.151-160.

[11] Horn, B.K.P. and Brooks, M.J. (1986) The Variational Approach to Shape from Shading, CVGIP, Vol. 33, No. 2, pp. 174-208.

[12] Koenderink, J.J. and van Doorn, A.J. (1979) The Internal Representation of Solid Shape with Respect to Vision, Biological Cybernetics, Vol. 32, pp. 211-216.

[13] Koenderink, J.J. and van Doorn, A.J. (1992) Surface Shape and Curvature Scales, IVC, Vol. 10, pp. 557-565.

[14] Koenderink, J.J., van Doorn, A.J. and Kappers, A.M.L. (1992) Surface Perception in Pictures, Perception and Psychophysics, Vol. 52, No. 5, pp. 487-496.

[15] Lin, S. and Lee, S.W. (1999) A Representation of Specular Appearance, ICCV, pp. 849-854.

[16] Lin, S. and Lee, S.W. (1999) Estimation of Diffuse and Specular Appearance, ICCV, pp. 855-860.

[17] Lin, S. and Lee, S.W. (2000) An Appearance Representation for Multiple Reflection Components, CVPR, pp. 105-110.

[18] Nayar, S.K., Ikeuchi, K. and Kanade, T. (1991) Surface Reflection: Physical and Geometrical Perspectives, PAMI, Vol. 13, No. 7, pp. 611-634.

[19] Nayar, S.K., Fang, X.S. and Boult, T.E. (1993) Removal of Specularities Using Color and Polarization, CVPR, pp. 583-590.

[20] Torrance, K. and Sparrow, E. (1967) Theory for Off-Specular Reflection from Roughened Surfaces, JOSA, Vol. 57, pp. 1105-1114.

[21] Wolff, L.B., Nayar, S.K. and Oren, M. (1998) Improved Diffuse Reflection Models for Computer Vision, IJCV, Vol. 30, No. 1, pp. 55-71.

[22] Worthington, P.L. and Hancock, E.R. (1999) New Constraints on Data-closeness and Needlemap consistency for SFS, PAMI, Vol. 21, No. 11, pp. 1250-1267. 

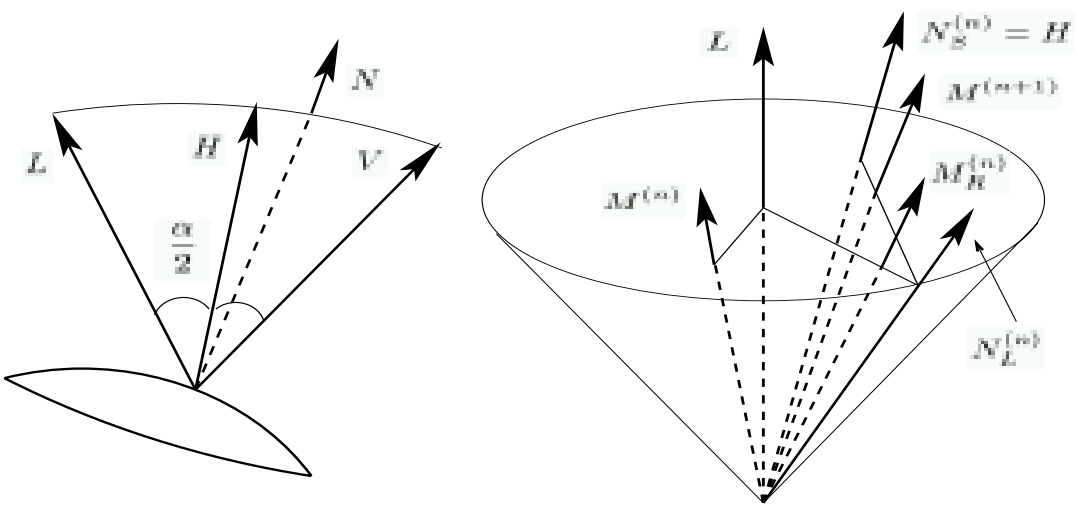

Figure 1: Geometrical representation of the new method
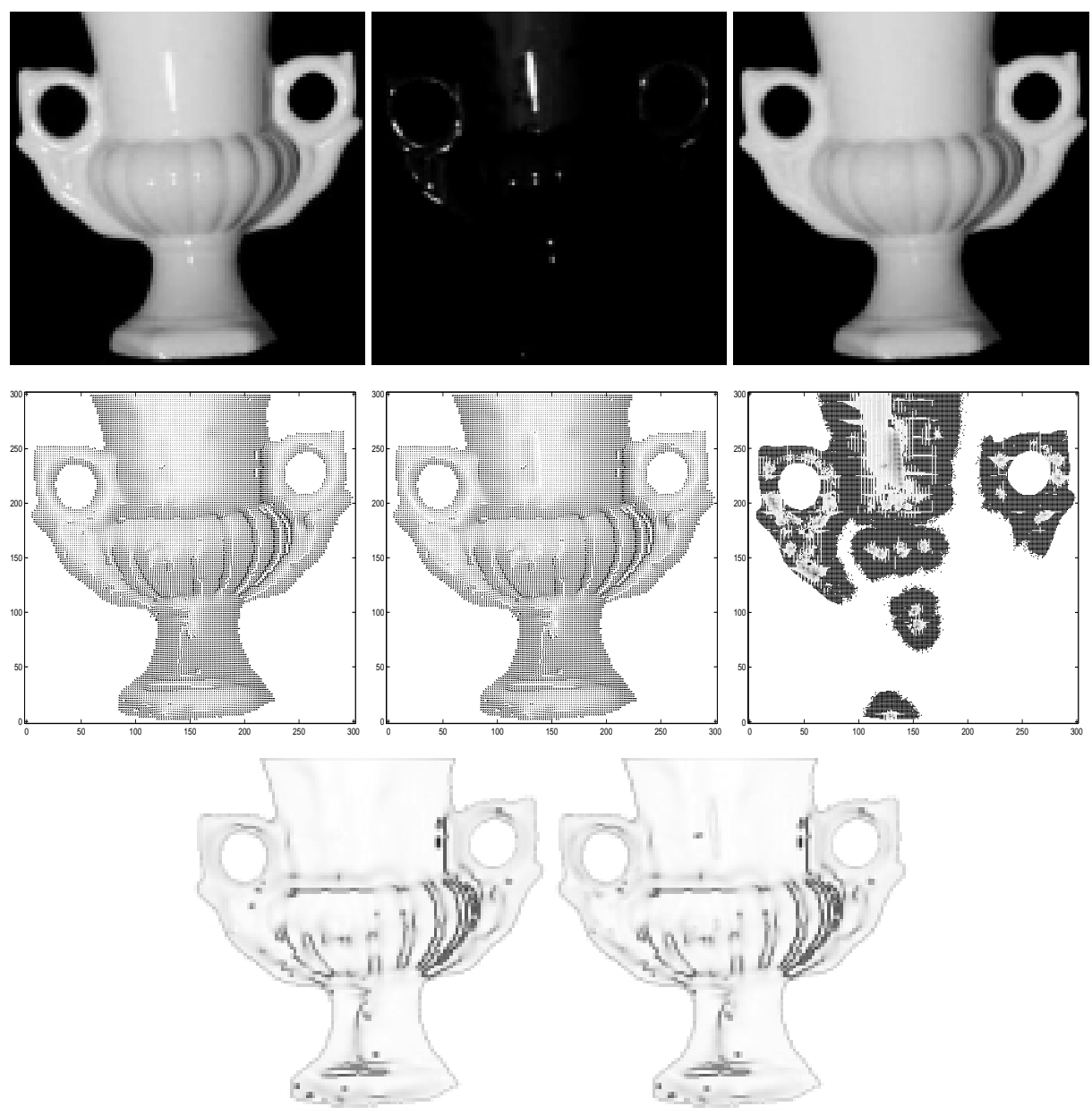

Figure 2: Results for a porcelain urn 


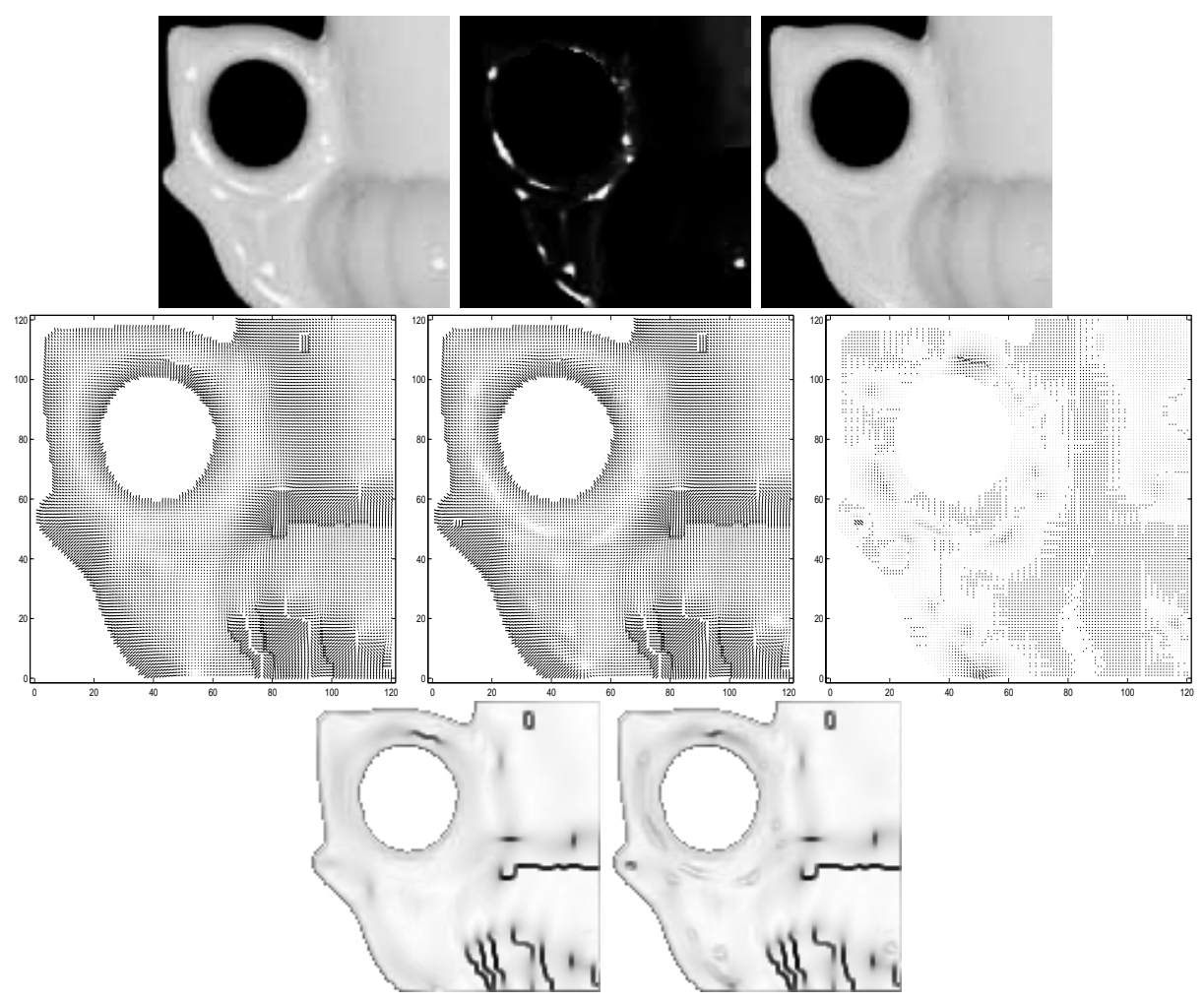

Figure 3: Results for the left handle of the urn

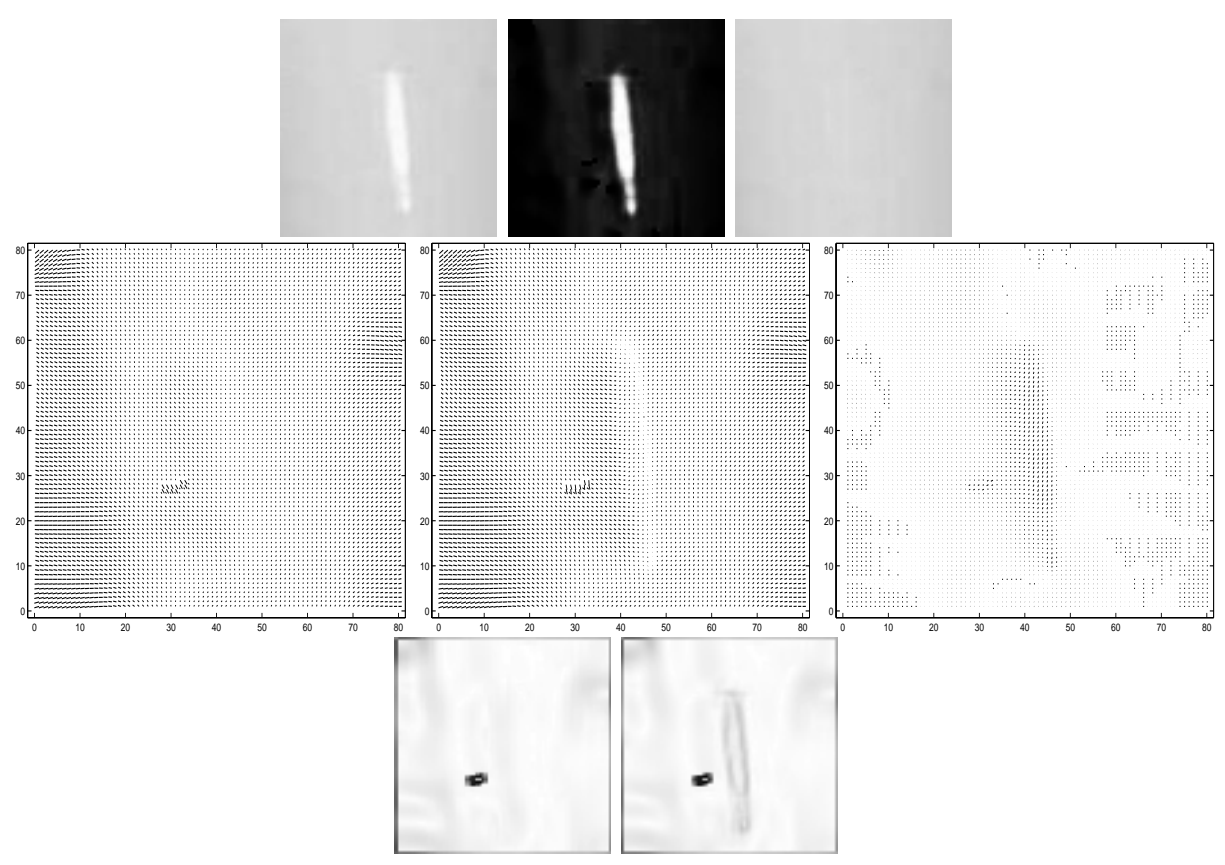

Figure 4: Results for the bright specular highlight of the urn 


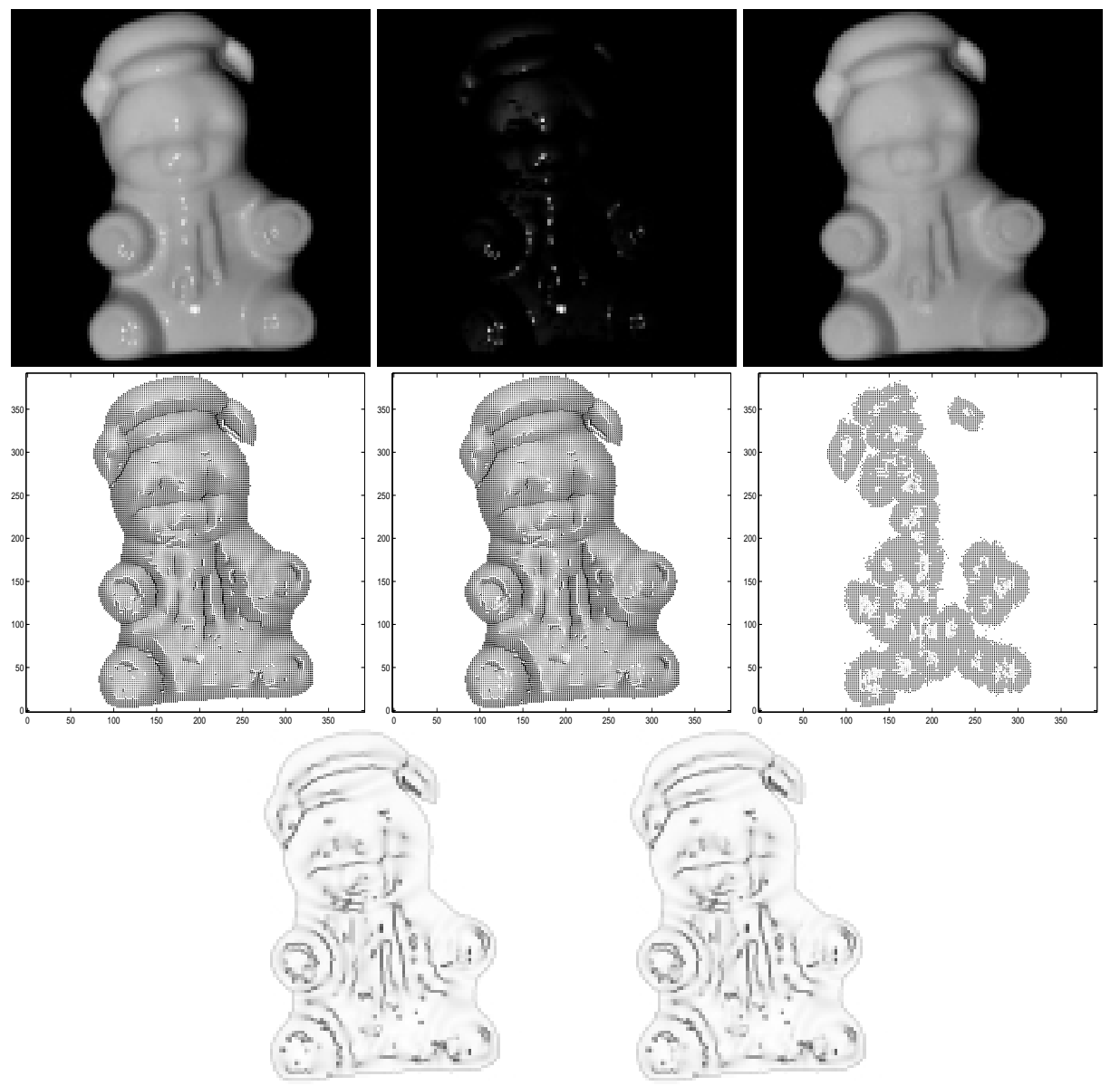

Figure 5: Results for a porcelain model of a bear

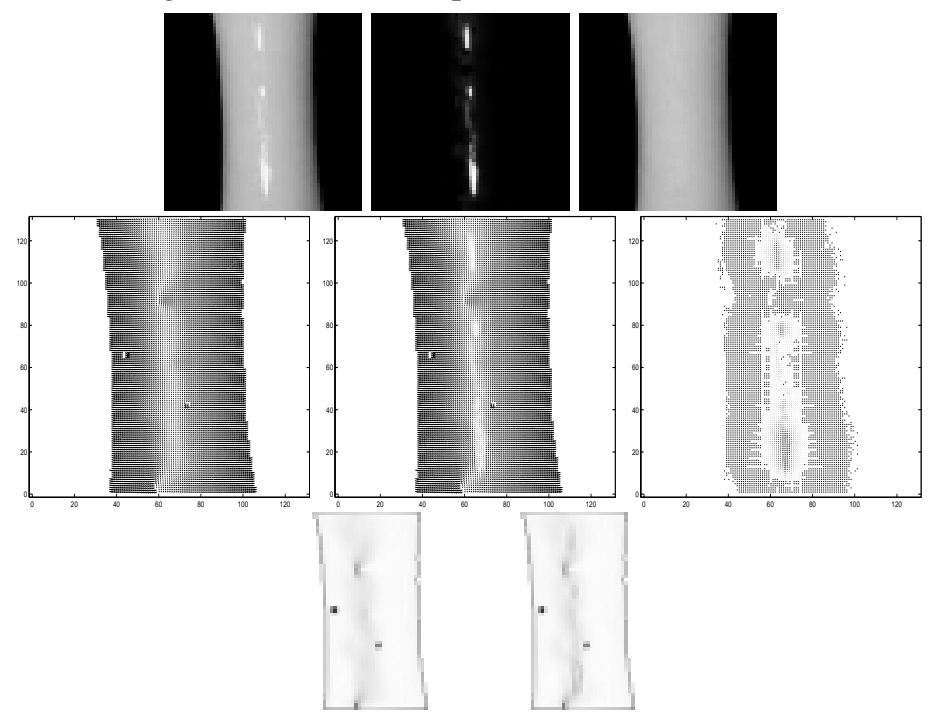

Figure 6: Results for the neck of a porcelain vase 